\title{
PNPLA3-Associated Steatohepatitis: Toward a Gene-Based Classification of Fatty Liver Disease
}

\author{
Marcin Krawczyk, MD ${ }^{1}$ Piero Portincasa, MD, $\mathrm{PhD}^{2}$ \\ ${ }^{1}$ Department of Medicine II, Saarland University Medical Center, \\ Homburg, Germany \\ 2 Department of Biomedical Sciences and Human Oncology, Clinica \\ Medica "A. Murri," University of Bari Medical School, Bari, Italy \\ Semin Liver Dis 2013;33:369-379.
}

Frank Lammert, MD, $\mathrm{PhD}^{1}$

\begin{abstract}
Address for correspondence Frank Lammert, MD, PhD, Department of Medicine II, Saarland University Hospital, Kirrberger Str. 100, 66421 Homburg, Germany (e-mail: frank.lammert@uks.eu).
\end{abstract}

\begin{abstract}
Keywords

- adiponutrin

- genomics

- genetic susceptibility

- nonalcoholic steatohepatitis

- steatosis

Nonalcoholic fatty liver disease is one of the most common hepatic disorders worldwide. Given the high-calorie nutrition of children and adults, nonalcoholic fatty liver disease (NAFLD) is expected to become a major cause of cirrhosis and eventually liver transplantation. Familial clustering and ethnic differences indicate that genetic factors contribute to NAFLD. Recently, the common variant p.I148M of the enzyme adiponutrin (PNPLA3) has emerged as a major genetic determinant of hepatic steatosis and nonalcoholic steatohepatitis as well as its pathobiological sequelae fibrosis, cirrhosis, and hepatocellular cancer. PNPLA3 encodes a lipid droplet-associated, carbohydrate-regulated lipogenic and/or lipolytic enzyme. Homozygous carriers of the PNPLA3 variant are prone to develop cirrhosis in the absence of other risk factors such as alcohol or viral hepatitis. Here we review the plethora of studies that unraveled the association between PNPLA3 and NAFLD in children and adults, discuss its distinct effects on liver and metabolic traits, and introduce the term PNPLA3-associated steatohepatitis (PASH) as a novel gene-based liver disease. Given the prevalence of the risk allele in 40 to $50 \%$ of Europeans, the authors conclude that PNPLA3 should be considered in the diagnostic workup of fatty liver disease and that homozygous risk allele carriers might benefit from careful cancer surveillance.
\end{abstract}

To date, fatty liver disease is one of the most common diseases worldwide. According to the latest surveys as much as $21 \%$ of adults in the United States may suffer from fatty liver diseasemore than 30 million patients nationwide. ${ }^{1}$ The most recent data on the burden of liver disease by the European Association for the Study of the Liver (EASL) indicate that nonalcoholic fatty liver disease (NAFLD) is emerging as the most common hepatic disorder and is likely to become a major cause for liver transplantation in Europe. Depending on the survey, the prevalence of NAFLD ranges from 2 to $44 \%$, but the prevalence of this condition among patients with type 2 diabetes can be as high as $70 \%{ }^{2}$ Often detected by abdominal ultrasonography in individuals without any apparent liver disease who do not consume excessive amounts of alcohol, fatty liver is often considered to be simply one of the "fellow travelers" of the

Issue Theme Lipids and the Liver; Guest Editor, David E. Cohen, MD, PhD obesity pandemics, given its epidemiological associations with metabolic syndrome and diabetes. However, an estimated 3 to $12 \%$ of the population suffer from the progressive form of NAFLD known as nonalcoholic steatohepatitis (NASH). ${ }^{3,4}$

According to the latest guidelines, ${ }^{5}$ the term nonalcoholic fatty liver disease covers several types of disorders, which may be benign or more severe in nature. In brief, NAFLD subdivides into nonalcoholic fatty liver (NAFL), nonalcoholic steatohepatitis (NASH), and NASH-cirrhosis. Nonalcoholic fatty liver disease comprises simple hepatic steatosis without any evidence of hepatic damage. According to current paradigms, patients with NAFL have only a small risk of liver disease progression. Nonalcoholic steatohepatitis in turn represents a progressive form of NAFLD characterized by chronic hepatocellular injury and inflammatory responses, which might
Copyright @ 2013 by Thieme Medical Publishers, Inc., 333 Seventh Avenue, New York, NY 10001, USA. Tel: +1(212) 584-4662. 
progress to liver fibrosis, cirrhosis, and hepatocellular cancer (HCC). If a patient with NASH develops liver cirrhosis, then this condition is termed NASH-cirrhosis. ${ }^{5}$ This classification implies that there are two major types of NAFLD: the most common benign NAFL type and a defined subgroup of patients with more severe lipid accumulation and inflammation, namely NASH. Indeed, patients with NASH are characterized by reduced overall life expectancy due to liver-related mortality, diabetes, and cardiovascular deaths, ${ }^{6}$ which is not generally observed in patients with steatosis. Hence, the early detection of individuals who are at increased risk of developing NASH is pivotal to prevent the progression of liver disease, and also to dissect the subgroup of patients at increased cardiovascular risk. A major problem, however, is that NASH remains asymptomatic for a long period in most patients. Given the lack of adequate imaging techniques and reliable noninvasive markers, ${ }^{7,8}$ liver biopsy is still formally required to detect or exclude the presence of NASH and to adequately grade and stage the liver condition in NAFLD patients.

Lately, a genetic predisposition caused by the frequent variant p.I148M of adiponutrin (PNPLA3) has been established as a strong genetic (i.e., noninvasive) hallmark of fatty liver disease even in the absence of environmental prosteatotic triggers. ${ }^{9}$ Moreover this variant has been associated not only with liver injury both in children and adults who suffer from hepatic steatosis, but also with steatosis and fibrosis in other chronic liver diseases. Here we present studies that established PNPLA3 as a genetic determinant of fatty liver, and we introduce the term PNPLA3-associated steatohepatitis $(P A S H)$ as a novel gene-based term for this type of fatty liver disease.

\section{PNPLA3 (Adiponutrin) Variant and Fatty Liver Disease}

Adiponutrin, the enzyme encoded by the PNPLA3 gene, is a 481-amino acid member of the patatin-like phospholipase domain-containing family (PNPLA). This domain was originally discovered in lipid hydrolases of potato and named after the most abundant protein of the potato tuber, patatin. However, because several family members are not phospholipases, a more appropriate gene symbol has been called for. ${ }^{10}$ PNPLA3 is expressed predominantly in the liver, skin, and adipose tissue. ${ }^{11}$ In a series of seminal genetic studies, the common nonsynonymous variant p.I148M $(\operatorname{rs738409}(>\mathrm{G}))$ of the PNPLA3 gene has emerged as the key genetic determinant of NAFLD in adults and pediatric patients. ${ }^{9}$ The first genome-wide association study (GWAS) in a large U.S.-based population comprising 2,111 individuals from different ethnic backgrounds demonstrated the p.I148M variant to be associated $\left(p=5.9 \times 10^{-10}\right)$ with increased liver fat content on a genome-wide significance level, as determined by ${ }^{1} \mathrm{H}-$ magnetic resonance spectrometry, irrespective of alcohol consumption, body mass index (BMI), and diabetes. ${ }^{12}$ This association was most prominent among patients of Hispanic descent, ${ }^{12}$ who are in general at a greater risk of developing fatty liver as compared with Caucasians and African
Americans. ${ }^{13-15}$ A subgroup analysis restricted to African Americans identified a second PNPLA3 variant (p.S453I, rs6006460, $G>T$ ), which is strongly associated with lower hepatic fat contents in this ethnic group. ${ }^{12}$

Interestingly, the common polymorphism p.I148M had been shown previously to correlate with serum activities of liver enzymes. In particular, the analyses of two large population-based cohorts with 12,419 and 61,089 participants, respectively, demonstrated that PNPLA3 polymorphisms are associated with serum alanine aminotransferase (ALT) and $\gamma$ glutamyl transpeptidase activities in healthy individua1s. ${ }^{16,17}$ The genetic association between the PNPLA3 mutation p.I148M and fatty liver disease was subsequently replicated in many studies. Kotronen et al ${ }^{18}$ investigated the effects of this variant on hepatic fat accumulation in Finnish individuals. In line with the results of the first GWAS, Finnish carriers of the risk allele were characterized by increased hepatic lipid contents (also quantified with proton magnetic resonance spectroscopy) irrespective of age, gender, or BMI. ${ }^{18}$ The PNPLA3 variant was also found to be strongly ( $p=4.3$ $\times 10^{-34}$ ) associated with hepatic lipid levels in a GWAS genotyping 2.4 million single-nucleotide polymorphisms (SNPs) in 7,176 individuals whose lipid contents in liver were quantified by another noninvasive method (computed tomography $[\mathrm{CT}]) .{ }^{19}$ Further studies showed that the PNPLA3 variant not only increases the odds of developing fatty liver itself, but it also determines the degree of hepatic injury and all histopathological aspects of NAFLD. A study of the histopathological hallmarks of NAFLD in 103 subjects from Argentina provided evidence that the PNPLA3 risk allele determines NAFLD severity. ${ }^{20}$ The comparison of genotype frequencies between individuals with simple steatosis $(N=40)$ and patients with NASH $(N=63)$ demonstrated an association between the risk genotype and histopathological disease severity, as assessed by liver biopsy (odds ratio, $[\mathrm{OR}]=1.9$ ). A similar analysis was performed in a cohort encompassing 574 Italian and English patients with NAFLD and 179 controls without fatty liver disease. ${ }^{21}$ Also in this study the frequencies of the PNPLA3 variant were associated with NAFLD severity as determined by liver biopsy. ${ }^{21}$ Here the authors identified not only a higher prevalence of the risk genotype among cases as compared with controls, but the PNPLA3 mutation was strongly associated with the presence of $\mathrm{NASH}$, steatosis grade $>1$, and fibrosis stage $>1$ independent of age, BMI, or diabetes. ${ }^{21}$ The detailed analysis of histopathological markers of NAFLD was performed by Rotman et al, ${ }^{22}$ demonstrating that the PNPLA3 variant is associated with portal $\left(p=2.5 \times 10^{-4}\right)$ and lobular $(p=0.005)$ inflammation, Mallory-Denk bodies $(p=0.02)$, and fibrosis $\left(p=7.7 \times 10^{-6}\right)$. A meta-analysis ${ }^{9}$ concluded that the PNPLA3 p.I148M variant is associated with increased risks for fatty liver (OR for homozygous carriers $=3.3$; OR for heterozygous carriers $=1.9$ ), NASH (OR for homozygous carriers $=3.1-3.3 ; \quad$ OR for heterozygous carriers $=2.7$ ), and fibrosis (OR for homozygous carriers $=3.3$; OR for heterozygous carriers $=2.1-2.4$ ) and most remarkably, the association with inflammation and fibrosis is independent of the severity of steatosis. 


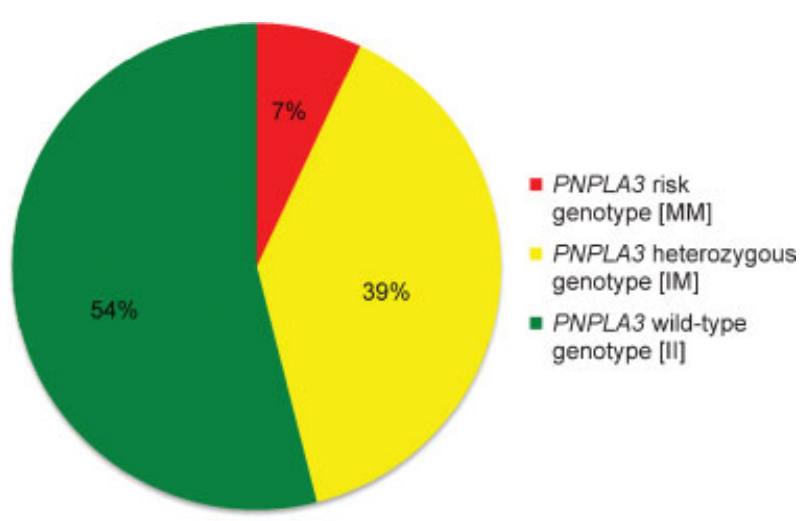

Fig. 1 Frequencies of the PNPLA3 p.I148M genotypes. According to our previous study in 899 individuals with chronic liver diseases, ${ }^{25}$ as many as 40 to $50 \%$ of European patients carry at least one copy of the rare allele $(\mathrm{M})$ that is associated with progressive liver diseases.

Recently, Anstee and coworkers from the European FLIP (Fatty Liver: Inhibition of Progression) Consortium ${ }^{23}$ have elegantly confirmed, genotyping candidate genes from association studies in a large cohort with histopathologically characterized liver disease, that the PNPLA3 variant is strongly associated with all histopathological features of NAFLD at genome-wide significance levels. Albeit other polymorphisms in the glucokinase regulator (GCKR) and tribbles homolog 1 (TRIB1) genes represented additional risk factors, only variant PNPLA3 conferred substantial and clinically relevant ORs (3.03.1) for the development of steatosis, its transition to NASH, and the progression of fibrosis. ${ }^{23}$ In contrast, the other genes identified represent only modifiers of subphenotypes with smaller effect sizes. ${ }^{24}$ According to our previous study, the risk allele is present in as many as 40 to $50 \%$ of Europeans (-Fig. 1). ${ }^{25}$ These findings have also been extended to other ethnic groups: Two recent Japanese histopathologically-based GWASs replicated the association with NAFLD severity and identified no other major susceptibility loci. ${ }^{26,27}$ Overall, the above studies summarized in -Table 1 document that the PNPLA3 mutation increases the risk of developing severe hepatic fat accumulation, progressive inflammation, and advanced fibrosis not only in Caucasian patients with NAFLD, but across different ethnicities.

\section{Association of the PNPLA3 Variant with Progressive Chronic Liver Diseases}

The PNPLA3 variant is associated not only with NAFLD, but also increases the odds of severe hepatic phenotypes in patients with other chronic liver diseases (-Table 2). In particular, the PNPLA3 risk allele p.148M is associated with increased inflammation and severe fibrosis as well as cirrhosis in patients with alcoholic liver disease. ${ }^{28-31}$ Similarly, carriers of this allele are also at risk for enhanced increased hepatic steatosis and fibrosis in the setting of chronic hepatitis $\mathrm{C}$ virus (HCV) infection, ${ }^{32-34}$ which can be further modulated by the amounts of the intraabdominal fat, ${ }^{35}$ and increased steatosis in chronic hepatitis B virus (HBV) infec- tion. ${ }^{36}$ Finally, in our previous analysis using transient elastography to quantify liver fibrosis in 899 patients with chronic liver diseases, we identified a prominent association between the PNPLA3 mutation and enhanced liver stiffness in a wide spectrum viral and nonviral chronic liver diseases. ${ }^{25}$ Sensitivity analysis showed that the association was present across a broad range of stiffness values $(12-40 \mathrm{kPa}),{ }^{25}$ indicating that the variant affects not only fibrogenesis, but also cirrhosis severity. In this line, once cirrhosis is present, carriers of the PNPLA3 mutation also have a 2- to 16 -fold increased risk of developing HCC (- Table 3). ${ }^{37-40}$ The homozygous p.148M genotype was found to predominantly increase the HCC risk in patients with alcoholic liver disease. ${ }^{40,41}$ In a series of newly diagnosed HCC cases, homozygosity for the genotype p.148M was even an independent risk factor for death. According to Hassan et al, ${ }^{39} \mathrm{HCC}$ patients with this PNPLA3 genotype displayed reduced median survival $(16.8 \mathrm{mo})$ in comparison to carriers of the wild-type allele (25.9 mo). Furthermore, a recent report by the European FLIP consortium showed that the cancer risk of homozygous PNPLA3 mutation carriers is 15-fold higher in comparison to the general population in the United Kingdom. $^{42}$

The latest reports investigated the association between PNPLA3 and liver status after transplantation. An early analysis of 176 subjects who were transplanted for HCV-cirrhosis did not identify any association between the recipient PNPLA3 genotype and the presence of advanced (F3) fibrosis (as determined by liver biopsy) 1,3 , and 5 years after transplantation. ${ }^{43}$ However, in this study the donor PNPLA3 genotypes were not determined. Finkenstedt et $\mathrm{al}^{44}$ in turn analyzed PNPLA3 genotype frequencies in both transplant recipients $(N=237)$ and donors $(N=255)$. Interestingly, homozygous carriers of the risk genotype were 14 times more likely to develop graft steatosis independently of other prosteatotic triggers (age, underlying disease, weight gain). ${ }^{44}$ Here, the donor genotype did not affect the development of graft steatosis, but further studies are required to dissect the specific effects of variant PNPLA3 after liver transplantation.

\section{Studies in Pediatric Cohorts}

Liver steatosis is emerging as an additional health problem in children as well. In line with the studies in adult patients, significant association between fatty liver and the PNPLA3 mutation was observed in children with NAFLD, too. In the study by Valenti et al, ${ }^{45} 149$ children with biopsy-proven NAFLD were included. The analysis of PNPLA3 genotypes in this cohort showed that the variant was not associated with adiposity, BMI, insulin resistance, lipid profile, or serum AST activities. ${ }^{45}$ However, the PNPLA3 variant determined the degree of hepatic steatosis. ${ }^{45}$ Moreover, children homozygous for the mutated allele were at highest risk of developing NASH: In the investigated cohort all children $(N=23)$ who were homozygous carriers of the PNPLA3 mutation were diagnosed with NASH. ${ }^{45}$ The association between the PNPLA3 mutation and pediatric NAFLD has also been detected in Caucasian and African American, ${ }^{46}$ Taiwanese, ${ }^{47}$ and to a 
Table 1 Selected studies investigating association of the PNPLA3 variant with hepatic and metabolic phenotypes (in chronological order)

\begin{tabular}{|c|c|c|c|c|c|}
\hline $\begin{array}{l}\text { Phenotypic } \\
\text { parameter }\end{array}$ & $\begin{array}{l}\text { Number of } \\
\text { individuals }\end{array}$ & $\begin{array}{l}\text { Population / } \\
\text { ethnicity }\end{array}$ & $P$ value & OR (95\% Cl) & Reference \\
\hline $\begin{array}{l}\text { Serum levels of liver } \\
\text { enzymes }{ }^{\mathrm{a}, \mathrm{b}}\end{array}$ & 12,419 & $\begin{array}{l}\text { Indian Asian, } \\
\text { European }\end{array}$ & $\begin{array}{l}\text { ALT } \\
p=8.4 \times 10^{-16}\end{array}$ & & 16 \\
\hline NAFLDa $\left.{ }^{1}{ }^{1} \mathrm{H}-\mathrm{MRS}\right)$ & 2,111 & $\begin{array}{l}\text { Hispanic, African } \\
\text { American, and Eu- } \\
\text { ropean American }\end{array}$ & $\begin{array}{l}\text { Steatosis } p=5.9 \\
\times 10^{-10} \\
\text { ALT } p=3.7 \times 10^{-4}\end{array}$ & & 12 \\
\hline NAFLD ( $\left.{ }^{1} \mathrm{H}-\mathrm{MRS}\right)$ & 291 & Finland & $\begin{array}{l}\text { Liver fat content } \\
p=0.011 \\
\text { AST } p=0.002\end{array}$ & & 18 \\
\hline NAFLD ( $\left.{ }^{1} \mathrm{H}-\mathrm{MRS}\right)$ & 330 & Germany & $\begin{array}{l}\text { Increased steatosis } \\
p=0.0001\end{array}$ & $2.38(1.37-4.20)$ & 58 \\
\hline $\begin{array}{l}\text { NAFLD (abdominal } \\
\text { ultrasonography, } \\
\text { liver biopsy) }\end{array}$ & $\begin{array}{l}172 \text { NAFLD } \\
94 \text { cases }\end{array}$ & $\begin{array}{l}\text { County } \\
\text { hospital of the city } \\
\text { of Buenos Aires }\end{array}$ & $\begin{array}{l}\text { NAFLD } \\
p<0.001\end{array}$ & $2.8(1.5-5.2)$ & 20 \\
\hline $\begin{array}{l}\text { Alcoholic liver dis- } \\
\text { ease (biochemical, } \\
\text { clinical assessment } \\
\text { and imaging) }\end{array}$ & $\begin{array}{l}434 \text { ALD } \\
482 \text { alcoholic } \\
\text { cirrhosis } \\
305 \text { controls }\end{array}$ & $\begin{array}{l}\text { Mestizos from } \\
\text { Mexico City }\end{array}$ & $\begin{array}{l}\text { Alcoholic cirrhosis } \\
p=1.7 \times 10^{-10}\end{array}$ & $2.25(1.74-2.90)$ & 28 \\
\hline NAFLD (liver biopsy) & $\begin{array}{l}574 \text { cases } \\
179 \text { controls }\end{array}$ & UK, Italy & $\begin{array}{l}\text { Steatosis grade }>1 \\
p=0.02 \\
\text { NASH } p=0.007 \\
\text { Fibrosis stage }>1 \\
p=0.01\end{array}$ & $\begin{array}{l}1.35(1.04-1.76) \\
1.50(1.12-2.04) \\
1.50(1.09-2.12)\end{array}$ & 21 \\
\hline $\begin{array}{l}\text { Serum aminotrans- } \\
\text { ferase activities }\end{array}$ & $\begin{array}{l}475 \text { overweight } \\
\text { children }\end{array}$ & Italy & $\begin{array}{l}\text { ALT } p=0.001 \\
\text { AST } p=0.022\end{array}$ & & 51 \\
\hline NAFLD (liver biopsy) & $\begin{array}{l}592 \text { cases } \\
1405 \text { controls }\end{array}$ & $\begin{array}{l}\text { European non- } \\
\text { Hispanic ancestry }\end{array}$ & $\begin{array}{l}\text { NAFLD } \\
p=3.6 \times 10^{-43}\end{array}$ & $3.26(2.11-7.21)$ & 59 \\
\hline NAFLD (liver biopsy) & $\begin{array}{l}149 \text { children and } \\
\text { adolescents }\end{array}$ & Italy & $\begin{array}{l}\text { Severity of steatosis } \\
p<0.0001 \\
\text { NASH } p<0.0001 \\
\text { Hepatocellular bal- } \\
\text { looning } p<0.0001 \\
\text { Lobular inflamma- } \\
\text { tion } p<0.0001 \\
\text { Fibrosis } p=0.01\end{array}$ & & 45 \\
\hline NAFLD (liver biopsy) & $\begin{array}{l}1,117 \text { cases ( } 894 \\
\text { adults, } 223 \\
\text { children) }\end{array}$ & USA & $\begin{array}{l}\text { Steatosis } p=0.03 \\
\text { Portal inflammation } \\
p=2.5 \times 10^{-4} \\
\text { Lobular inflamma- } \\
\text { tion } p=0.005 \\
\text { Mallory-Denk } \\
\text { bodies } p=0.015 \\
\text { Fibrosis } p=7.7 \\
\times 10^{-6} \\
\text { NAS } p=0.004\end{array}$ & $\begin{array}{l}1.46(1.07-2.01) \\
1.57(1.24-1.99) \\
1.84(1.33-2.55) \\
1.60(1.46-3.07)\end{array}$ & 22 \\
\hline $\begin{array}{l}\text { NAFLD (MRI, liver } \\
\text { biopsy) }\end{array}$ & $\begin{array}{l}85 \text { obese children } \\
\text { and adolescents }\end{array}$ & $\begin{array}{l}\text { Caucasian, African } \\
\text { American, Hispanic }\end{array}$ & $\begin{array}{l}\text { NAFLD Caucasians } \\
p=3.6 \times 10^{-4} \\
\text { NAFLD African } \\
\text { Americans } \\
p=0.012 \\
\text { NAFLD Hispanics } \\
p=0.52\end{array}$ & & 46 \\
\hline NAFLD ( $\left.{ }^{1} \mathrm{H}-\mathrm{MRS}\right)$ & $\begin{array}{l}218 \text { diabetic } \\
\text { patients }\end{array}$ & France & Steatosis $p=0.04$ & & 77 \\
\hline
\end{tabular}


Table 1 (Continued)

\begin{tabular}{|c|c|c|c|c|c|}
\hline $\begin{array}{l}\text { Phenotypic } \\
\text { parameter }\end{array}$ & $\begin{array}{l}\text { Number of } \\
\text { individuals }\end{array}$ & $\begin{array}{l}\text { Population / } \\
\text { ethnicity }\end{array}$ & $P$ value & OR (95\% CI) & Reference \\
\hline NAFLD (MRI) & 327 & Hispanics & $\begin{array}{l}\text { Steatosis } \\
p<0.0001 \\
\text { Lower HDL } p=0.03\end{array}$ & & 78 \\
\hline $\begin{array}{l}\text { NAFLD (abdominal } \\
\text { sonography) }\end{array}$ & 520 obese children & Taiwan & $\begin{array}{l}\text { Steatosis } \\
p<0.0001 \\
\text { Increased ALT } \\
p<0.0001\end{array}$ & $\begin{array}{l}2.96(1.57-5.59) \\
5.84(2.59-13.16)\end{array}$ & 47 \\
\hline $\begin{array}{l}\text { Liver stiffness (tran- } \\
\text { sient elastography) }\end{array}$ & 899 & Germany & $\begin{array}{l}\text { TE }>13.0[\mathrm{kPa}] \\
p=0.005\end{array}$ & $1.56(1.14-2.14)$ & 25 \\
\hline $\begin{array}{l}\text { Liver steatosis in } \\
\text { patients with non-3 } \\
\text { HCV (liver biopsy) }\end{array}$ & 626 & Caucasian & $p<0.001$ & $1.9(1.6-2.3)$ & 32 \\
\hline $\begin{array}{l}\text { Alcoholic liver dis- } \\
\text { ease (liver biopsy) }\end{array}$ & 1,043 & Germany & $\begin{array}{l}\text { Alcoholic cirrhosis } p \\
=1.2 \times 10^{-5} \\
\text { Increased ALT } \\
p=0.0042\end{array}$ & $\begin{array}{l}2.79(1.55-5.04) \\
2.33(1.27-4.26)\end{array}$ & 29 \\
\hline $\begin{array}{l}\text { Steatosis, liver } \\
\text { fibrosis and HCC in } \\
\text { HCV } \\
\text { (liver biopsy) }\end{array}$ & $\begin{array}{l}819 \text { HCV } \\
261 \text { NAFLD } \\
179 \text { controls }\end{array}$ & Italy & $\begin{array}{l}\text { Steatosis } p<0.001 \\
\text { Cirrhosis } p=0.002 \\
\text { Treatment response } \\
p=0.006 \\
\text { HCC occurrence } \\
p=0.002\end{array}$ & $\begin{array}{l}1.90(1.4-2.7) \\
1.47(1.2-1.9) \\
0.63(0.4-0.8) \\
\\
2.16(1.3-3.6)\end{array}$ & 33 \\
\hline $\begin{array}{l}\text { Alcoholic liver dis- } \\
\text { ease (liver biopsy) }\end{array}$ & $\begin{array}{l}330 \text { cases } \\
328 \text { controls }\end{array}$ & $\begin{array}{l}\text { Caucasian } \\
\text { Europeans }\end{array}$ & $\begin{array}{l}\text { ALD } p=0.008 \\
\text { Steatosis } p=0.048 \\
\text { Fibrosis } p=0.001 \\
\text { Cirrhosis } p=0.02\end{array}$ & $\begin{array}{l}1.54(1.12-2.11) \\
2.08(1.15-3.77)\end{array}$ & 30 \\
\hline $\begin{array}{l}\text { NAFLDa (CT, liver } \\
\text { biopsy) }\end{array}$ & $\begin{array}{l}7,176 \text { - СТ } \\
592 \text { - biopsied cases } \\
1,405 \text { - Controls }\end{array}$ & Iceland, USA & $\begin{array}{l}\text { CT-assessed steato- } \\
\text { sis } p=4.3 \times 10^{-34} \\
\text { Biopsy-proven } \\
\text { NAFLD } \\
p=3.6 \times 10^{-43}\end{array}$ & & 19 \\
\hline $\begin{array}{l}\text { Steatosis and fibro- } \\
\text { sis in HCV (liver } \\
\text { biopsy) }\end{array}$ & 537 & $\begin{array}{l}\text { Caucasian: Belgium, } \\
\text { Germany, France }\end{array}$ & $\begin{array}{l}\text { Steatosis } p=0.034 \\
\text { Fibrosis } p=0.002 \\
\text { Fibrosis progression } \\
p=0.013\end{array}$ & $\begin{array}{l}2.55(1.08-6.03) \\
3.13(1.50-6.51) \\
2.64(1.22-5.67)\end{array}$ & 34 \\
\hline $\begin{array}{l}\text { Serum glucose } \\
\text { levels }\end{array}$ & 487 & Romania & $\begin{array}{l}\text { Increased serum } \\
\text { glucose } p=0.0001\end{array}$ & & 54 \\
\hline Liver density (CT) & 422 cases with T2D & African American & $\begin{array}{l}\text { Liver density } \\
p=0.0075 \\
\text { Steatosis } p=0.035\end{array}$ & & 79 \\
\hline $\begin{array}{l}\text { Concentrations of } \\
\text { liver enzymes in } \\
\text { plasma }\end{array}$ & 61,089 & $\begin{array}{l}\text { Caucasian, Indian } \\
\text { Asian }\end{array}$ & $\begin{array}{l}\text { ALT } \\
p=1.2 \times 10^{-45}\end{array}$ & & 17 \\
\hline $\begin{array}{l}\text { NAFLDa (liver } \\
\text { biopsy) }\end{array}$ & $\begin{array}{l}529 \text { cases } \\
932 \text { controls }\end{array}$ & Japan & $\begin{array}{l}\text { NAFLD } p=1.4 \\
\times 10^{-10} \\
\text { NASH } \\
p=1.7 \times 10^{-16}\end{array}$ & $\begin{array}{l}1.66(1.43-1.94) \\
2.18(1.81-2.63)\end{array}$ & 26 \\
\hline $\begin{array}{l}\text { Serum triglyceride } \\
\text { levels }\end{array}$ & 18,921 & Sweden, Scotland & $\begin{array}{l}\text { T2D risk } p=0.04 \\
\text { T2D risk } p=0.001 \\
\text { (severely obese) }\end{array}$ & $\begin{array}{l}1.09(1.01-1.39) \\
1.37(1.13-1.66)\end{array}$ & 56 \\
\hline $\begin{array}{l}\text { Serum cholesterol } \\
\text { and triglycerides }\end{array}$ & 5,847 & Denmark & $\begin{array}{l}\text { Decreased TG in IGR } \\
p=5.1 \times 10^{-5} \\
\text { Decreased choles- } \\
\text { terol in IGR } \\
p=1.5 \times 10^{-4}\end{array}$ & & 53 \\
\hline
\end{tabular}


Table 1 (Continued)

\begin{tabular}{|c|c|c|c|c|c|}
\hline $\begin{array}{l}\text { Phenotypic } \\
\text { parameter }\end{array}$ & $\begin{array}{l}\text { Number of } \\
\text { individuals }\end{array}$ & $\begin{array}{l}\text { Population / } \\
\text { ethnicity }\end{array}$ & $P$ value & OR $(95 \% \mathrm{Cl})$ & Reference \\
\hline HCC prognosis & 638 & Japan & $\begin{array}{l}\text { Poor prognosis in } \\
\text { ALD patients with } \\
\text { low BMI and HCC } \\
p=0.028\end{array}$ & & 41 \\
\hline $\begin{array}{l}\text { Insulin resistance } \\
\text { and viral load in HCV } \\
\text { (genotype 2) } \\
\text { patients }\end{array}$ & 308 & $\begin{array}{l}\text { Denmark, Finland, } \\
\text { Norway, Sweden }\end{array}$ & $\begin{array}{l}\text { Insulin resistance } \\
p=0.023 \\
\text { Lower viral load } \\
p=0.005\end{array}$ & & 55 \\
\hline $\begin{array}{l}\text { NAFLD (abdominal } \\
\text { sonography) }\end{array}$ & $\begin{array}{l}203 \text { cases } \\
202 \text { controls }\end{array}$ & China & $\begin{array}{l}\text { NAFLD } \\
p=7.6 \times 10^{-8}\end{array}$ & & 80 \\
\hline $\begin{array}{l}\text { ALT levels in obese } \\
\text { children }\end{array}$ & 1,037 & Mexico & $\begin{array}{l}\text { Elevated ALT } \\
p=3.710^{-8}\end{array}$ & $3.7(2.3-5.9)$ & 48 \\
\hline $\begin{array}{l}\text { NAFLDa (liver biop- } \\
\text { sy, CT, MRI) }\end{array}$ & $\begin{array}{l}564 \text { cases } \\
1,946 \text { controls }\end{array}$ & Japan & $\begin{array}{l}\text { NAFLD } \\
p=6.8 \times 10^{-14}\end{array}$ & 2.05 & 27 \\
\hline $\begin{array}{l}\text { Steatosis in HBV } \\
\text { (liver biopsy) }\end{array}$ & 235 & Italy & $\begin{array}{l}\text { Steatosis } p=0.05 \\
\text { Severe steatosis } \\
p=0.011 \\
\text { NAS }>2 p=0.023\end{array}$ & $\begin{array}{l}1.62(1.00-7.0) \\
6.03(1.23-5.0) \\
1.70(1.07-2.74)\end{array}$ & 81 \\
\hline HCC risk & $\begin{array}{l}257 \text { cases } \\
494 \text { controls }\end{array}$ & Caucasian & $\begin{array}{l}\mathrm{HCC} p<0.001 \\
\text { Cirrhosis P } 0.039 \\
\text { Death P } 0.004\end{array}$ & $\begin{array}{l}3.21(1.68-6.41) \\
2.48(1.05-5.87) \\
\text { HR 2.11 (1.26-3.52) }\end{array}$ & 39 \\
\hline
\end{tabular}

Abbreviations: ALD, alcoholic liver disease; ALT, alanine aminotrasferase; AST, aspartate transaminase; CT, computed tomography; HCC, hepatocellular carcinoma; HCV, hepatitis C virus; IGR, impaired glucose regulation; kPa, kilopascal; MRI, magnetic resonance imaging, MRS; magnetic resonance spectroscopy; NAFLD, nonalcoholic fatty liver disease; NAS, NAFLD activity score; NASH, nonalcoholic steatohepatitis; T2D, type 2 diabetes; TE, transient elastography.

${ }^{a}$ GWAS design.

bStudy investigating the PNPLA3 SNP rs2281135.

limited extent, Hispanic ${ }^{46}$ obese children. Interestingly, children carrying the PNPLA3 risk allele seem to be predisposed to an early development of NAFLD. ${ }^{22}$ The latest analysis of 6 to 12-year-old Mexican children showed that already at this age the PNPLA3 mutation may be associated with increased serum ALT activities, ${ }^{48}$ and we detected the same association

Table 2 Key examples of liver diseases associated with variant PNPLA3

\begin{tabular}{|l|l|l|}
\hline Disease & Study & Year \\
\hline Nonalcoholic fatty liver disease & ${\text { Romeo et } \text { al }^{12}}^{2}$ & 2008 \\
\hline Alcoholic liver cirrhosis & ${\text { Tian et } \text { al }^{28}}^{2010}$ \\
\hline & Stickel $^{29}$ & 2011 \\
\hline Liver fibrosis & Krawczyk et al $^{25}$ & 2011 \\
\hline HBV steatosis & ${\text { Vigano et } \text { al }^{36}}^{2013}$ \\
\hline HCV steatosis & Cai et al $^{32}$ & 2011 \\
\hline Alcohol and HCV cirrhosis & Müller et al & 2011 \\
\hline HCV cirrhosis & Valenti et al $^{33}$ & 2011 \\
\hline Hepatocellular cancer & Nischalke et al & 2011 \\
\hline
\end{tabular}

Abbreviations: HBV, hepatitis B virus; HCV, hepatitis C virus. in a cohort of German children aged 5 to 9 years. ${ }^{49} \mathrm{~A}$ practical consequence is that weight loss might substantially improve the liver status in children carrying the risk variant and rescue the deleterious PNPLA3-associated liver phenotype. ${ }^{50}$ This possibility points to the need for early detection of pediatric patients carrying this mutation who require more careful follow-up and tailored therapies aiming at weight loss and physical activity. ${ }^{51}$

\section{Variant PNPLA3 and Metabolic Traits}

In general, patients with NAFLD often present with pathological lipid and glucose homeostasis. ${ }^{52}$ However, an association of PNPLA3 with metabolic traits in humans remains controversial. Interestingly, the effects of variant PNPLA3 on metabolic characteristics might be different in individuals with normal and impaired glucose tolerance. A Danish analysis of more than 4,000 individuals with normal glucose tolerance demonstrated a potential association of the risk allele with increased fasting glucose levels $(p=0.04)$, but the same allele was associated with lower serum levels of triglycerides and cholesterol in patients with impaired glucose intolerance $(N=1,357){ }^{53}$ In line with these results, we ${ }^{54}$ and other groups ${ }^{55,56}$ identified a possible association between glucose metabolism and the PNPLA3 p.I148M mutation. It has also 
Table 3 Studies reporting an association between variant PNPLA3 and HCC

\begin{tabular}{|c|c|c|c|c|c|c|}
\hline Study & Design & Setting & Etiology & $N$ (HCC: cirrhosis) & OR & $95 \% \mathrm{Cl}$ \\
\hline Valenti et $\mathrm{al}^{33}$ & Retrospective & HCV patients & $\mathrm{HCV}$ & 50: 275 & 2.2 & $1.3-2.6$ \\
\hline Ginanni Corradini et al $\left.\right|^{82}$ & Retrospective & Cirrhotic patients & $\mathrm{HCV}$ & 90: 131 & 2.2 & $1.4-3.5$ \\
\hline Nischalke et al ${ }^{40}$ & Retrospective & Cirrhotic patients & $\begin{array}{l}\text { HCV } \\
\text { Alcohol }\end{array}$ & $\begin{array}{l}\text { 80: } 80 \\
81: 81\end{array}$ & $\begin{array}{l}1.7 \\
2.8\end{array}$ & $\begin{array}{l}0.5-5.3 \\
1.6-6.4\end{array}$ \\
\hline Falleti et al ${ }^{83}$ & Retrospective & Cirrhotic patients & Mixed & 141: 342 & 1.8 & $1.1-2.9$ \\
\hline Trepo et $\mathrm{al}^{37}$ & Retrospective & Cirrhotic patients & Alcohol & 145: 426 & 4.7 & $2.6-8.4$ \\
\hline Burza et al $^{38}$ & Prospective & Swedish Obese Subjects Study & Obesity & 407 & 16.0 & $2.3-111$ \\
\hline Guyot et al ${ }^{84}$ & Prospective & Cirrhotic patients & $\begin{array}{l}\text { HCV } \\
\text { Alcohol }\end{array}$ & $\begin{array}{l}93: 160 \\
66: 213\end{array}$ & $\begin{array}{l}1.0 \\
1.9\end{array}$ & $\begin{array}{l}0.6-1.9 \\
1.3-2.8\end{array}$ \\
\hline Hassan et $a^{39}$ & Case-control & Cirrhotic patients & Mixed & 257: 494 controls & 3.2 & $1.7-6.4$ \\
\hline
\end{tabular}

Abbreviations: $\mathrm{Cl}$, confidence interval; $\mathrm{HCC}$, hepatocellular carcinoma; $\mathrm{HCV}$, hepatitis $\mathrm{C}$ virus; OR, odds ratio. Source: Adapted and modified from Valenti et al. ${ }^{74}$

been reported that the PNPLA3 variant results in decreased hepatic very low-density lipoprotein (VLDL) secretion, which would further contribute to increased lipid accumulation in liver. ${ }^{57}$ However, several studies did not show any relationship between this mutation and HOMA index, serum glucose, or lipid levels. ${ }^{19,45,58,59}$ These results contradict the prevailing paradigm that insulin resistance represents the main driver of common NAFLD. ${ }^{60}$ Indeed, a dissociation between the presence of fatty liver and insulin resistance appears to be present among carriers of the PNPLA3 risk variant. ${ }^{58}$ Moreover, carriers of the rare allele develop fatty liver irrespective of their BMI. ${ }^{21,22,59}$ Hence, PNPLA3-associated fatty liver represents an example that excessive deposition of fat in the liver is not the cause of hepatic insulin resistance, pointing to the more complex relationships between steatosis and insulin resistance. ${ }^{61}$ Vice versa, the effect of PNPLA3 variation on metabolic traits does not seem to be the driving force of hepatic fat accumulation, and carriers of the risk variant may develop severe hepatic steatosis even in the absence of disrupted systemic glucose and/or lipid homeostasis.

\section{Functional Analyses of the PNPLA3 Variant}

All the above studies established the PNPLA3 mutation p. I148M as a common genetic marker of NAFLD and triggered studies to unravel the functional consequences of the variant. The close similarity to adipose triglyceride lipase and the presence of typical structural motifs $(\alpha-\beta-\alpha$ sandwich structure, GXSXG motif within a catalytic dyad) suggested a lipase function for PNPLA3. ${ }^{10}$ Chen et al ${ }^{62}$ generated a Pnpla3knockout mouse and investigated metabolic traits and lipid contents of livers from these mice. Interestingly, loss of Pnpla3 in mice neither affected hepatic lipid composition nor serum activities of liver enzymes under normal or high-fat diets. ${ }^{62}$ Moreover, knocking-out Pnpla3 apparently did not have any effects on body fat composition and metabolic markers, in particular insulin sensitivity or glucose levels. ${ }^{62}$ Comparable results were provided by Basantani et $\mathrm{al}^{63}$ who also investi- gated metabolic and hepatic phenotypes in Pnpla3 $^{-1-}$ mice and did not detect any specific phenotypes that could be related to PNPLA3 deficiency.

Because it became apparent that loss of the PNPLA3 function is not a driver of the hepatic phenotype in individuals carrying the p.I148M variant and that knockout mice do not provide related phenotypes, the variant was studied further in vitro. ${ }^{64,65}$ The expression of PNPLA3 was shown to be regulated by carbohydrates, via sterol regulatory element binding protein-1c (SREBP1c) as well as specific fatty acids. ${ }^{11}$ Initially, the p.I148M substitution was demonstrated to reduce the lipolytic activity (but only modestly the substrate affinity) of recombinant PNPLA3. ${ }^{65}$ Kumari et al ${ }^{64}$ demonstrated that overexpression of PNPAL3 in various cell lines enhances intracellular diacylglycerol and phospholipid synthesis. Subsequently, the p.148M mutant was generated by site-directed mutagenesis, purified in Escherichia coli, and incubated with radiolabeled substrates. ${ }^{64}$ Analysis by thinlayer chromatography demonstrated that the purified enzyme enhances the acyl-CoA-dependent acylation of lysophosphatidic acid (LPA) to generate phosphatidic acid, i.e. possesses LPA acyltransferase activity. ${ }^{64}$ This activity of the mutated enzyme was in turn 2.0-fold higher as compared with the wild-type form, indicating that the amino acid substitution p.I148M leads to a gain of function of the enzyme. ${ }^{64}$ These findings suggested that PNPLA3 might represent an enzyme that metabolizes LPA to phosphatidic acid, which can subsequently be used in the synthesis of triglycerides. Further studies ${ }^{66}$ performed in transgenic mice overexpressing Pnpla3 in liver demonstrated that these animals develop fatty liver due to triacylglycerol accumulation as well as several alterations of hepatic lipid metabolism (i.e., increased synthesis of fatty acids and triacylglycerol, impaired hydrolysis of triglycerides, depletion of long-chain polyunsaturated fatty acids). ${ }^{66}$ Indeed in hepatocytes, most of the lipids are stored within lipid droplets, and livers from NAFLD patients are characterized by the increased number and size of lipid droplets within hepatocytes. Of note, PNPLA3 
is predominantly localized to the membrane- and lipid droplet-associated cellular fractions. ${ }^{67}$ Apparently, in carriers of the p.148M mutation, modulation of PNPLA3-associated pathways might increase the size of the lipid droplets. ${ }^{68}$ The mice overexpressing Pnpla3 were characterized by enhanced hydrolysis of triglycerides and increased formation of fatty acids and triglycerides, which all point to the fact that the PNPLA3 variant leads to numerous aberrations in intrahepatic lipid homeostasis and remodeling of hepatic lipid droplets. ${ }^{66}$

\section{PASH: PNPLA3-Associated Steatohepatitis and Future Directions}

Nonalcoholic fatty liver disease gains growing acceptance as a potentially severe chronic liver disease among hepatologists in particular and physicians in general. ${ }^{69}$ On the other hand, the diagnosis of NAFLD and its subtypes is still challenging in clinical practice. Fatty liver disease is usually diagnosed in individuals who present with a typical "bright liver" image on abdominal ultrasonography do not consume excessive amounts of alcohol (i.e. less than $20-30 \mathrm{~g} / \mathrm{d}$ ), and do not suffer from other specific liver diseases. ${ }^{70}$ In addition to ultrasound, which can be used as a screening tool, other noninvasive methods for quantifying hepatic fat contents have been developed; however, there is still a need for further evaluation. ${ }^{71}$ Given the studies reviewed here ( - Table 1), PNPLA3 genotyping may be used as a novel noninvasive marker for an increased risk of progressive fatty liver disease and could be included in the clinical decision making in patients with chronic liver diseases. So far, several subtypes of steatohepatitis are known by familiar acronyms, in particular $\mathrm{NASH}^{72}$ and alcoholic steatohepatitis (ASH). More recently, BASH (both alcoholic and nonalcoholic steatohepatitis), CASH (chemotherapy-associated steatohepatitis), and DASH (drug-associated steatohepatitis) have been suggested to be added to the etiologyoriented inventory of steatohepatitis subtypes. Of note, such conditions are caused by definable environmental prosteatotic triggers. Because in patients who carry the PNPLA3 risk variant increased lipid contents and inflammation in liver can be driven exclusively by PNPLA3 in the absence of environmental risk factors, we propose "PASH" (i.e., PNPLA3-associated steatohepatitis) as a novel gene-based liver disease entity (-Fig. 2). PNPLA3-associated steatohepatitis represents an example how to (re)classify disease according to molecular pathways and pathophysiological changes in the era of personalized medicine. ${ }^{73}$ As of now, we suggest to diagnose PASH in the subgroup of patients with steatohepatitis who are homozygous carriers of the risk allele and who do not present other risk factors for fatty liver disease such as alcohol abuse and metabolic syndrome, or chronic viral hepatitis. -Fig. 2 summarizes the spectrum of PNPLA3-related liver phenotypes.

The new gene-based disease classification might have clinical implications that need to be tested in prospective clinical studies. Indeed, carriers of the PNPLA3 risk allele could benefit from a more systematic, early and careful surveillance of complications of progressive fatty liver disease, including HCC in the absence of cirrhosis. ${ }^{74}$ Although to date prospective studies concerning long-term effects of this variant on

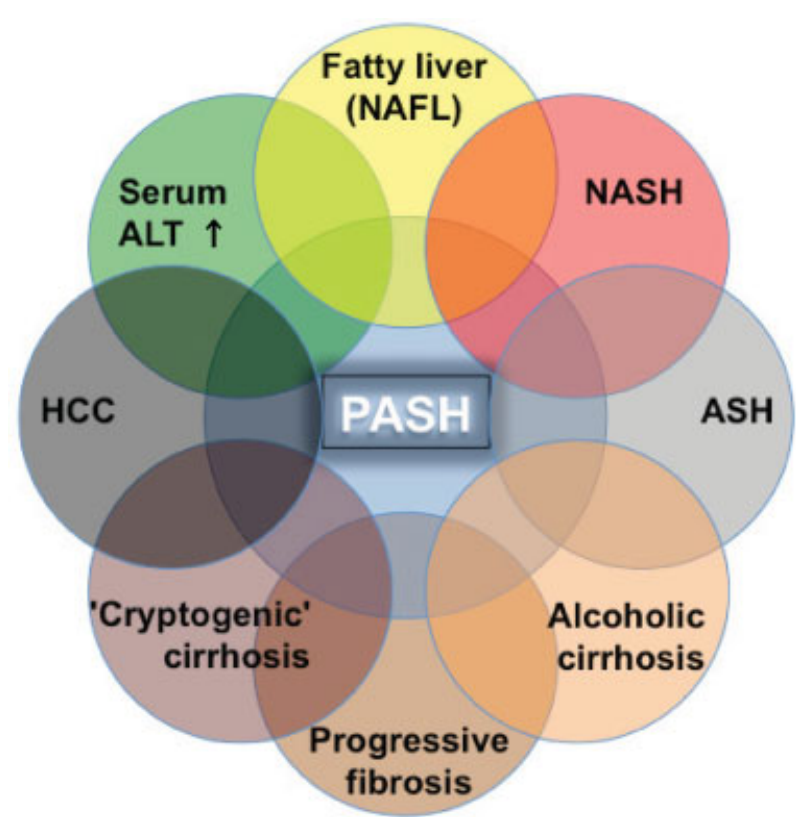

Fig. 2 PNPLA3-associated steatohepatitis (PASH) phenotypes associated with variant PNPLA3. Carriers of the p.148M allele are at increased risk of severe hepatic phenotypes, ranging from excessive hepatic fat accumulation and steatohepatitis to progressive fibrosis and HCC. ALT, alanine aminotransferase; ASH, alcoholic steatohepatitis, HCC, hepatocellular carcinoma; NAFL, nonalcoholic fatty liver disease; NASH, nonalcoholic steatohepatitis.

liver status are lacking, investigations in small groups of patients underscore the notion that weight loss may have beneficial effects on the liver status in the carriers of the rare allele. ${ }^{50,75}$ Although fatty liver disease is often associated with the metabolic syndrome, the PASH subtype does not seem to be substantially associated with the nonhepatic manifestations of this syndrome (body mass index, insulin resistance, dyslipidemia). As delineated above, the presence of the PNPLA3 risk allele is predominantly associated with liver phenotypes. As a result, we speculate that patients with this prosteatotic variant might easily be overlooked using our conventional connotations in clinical practice. On the other hand, because the frequency of the PNPLA3 risk allele is $\sim 20 \%$ of Europeans (- Fig. 1), 21,27,30 it might even be incorporated in the routine workup of patients with chronic liver diseases of unknown etiology. The variant could also be also used in future intervention studies. Indeed, at the moment we are still in need of evidence-based therapeutic strategies that lower hepatic fat content. ${ }^{7,76}$ Although several compounds have been tested, the latest American Association for the Study of Liver Diseases and American Gastroenterological Association guidelines ${ }^{5}$ recommend, as before, lifestyle interventions and vitamin $\mathrm{E}$ in nondiabetic adults with biopsy-proven NASH. ${ }^{5}$ Future interventions with randomization of patients according to the PNPLA3 genotype might result in precise therapy for carriers of the prosteatotic genotype. In patients with PNPLA3-associated steatohepatitis the design and evaluation of gene-based preventive and therapeutic approaches to a common liver disease, once 
thought to be a visionary promise, is closer to clinical practice than ever before.

\section{Acknowledgments}

This review is based, in part, on a concept developed by FL for the $49^{\text {th }}$ Annual Meeting of the European Society for Clinical Investigation, April 2013, and published, in part, as an abstract in Eur J Clin Invest 2013; 43 (Suppl. 1): 66. Experimental work related to this review was supported by the Deutsche Forschungsgemeinschaft (SFB/TRR 57 TP 01 and LA997/7-1 to FL) and Saarland University (HOMFOR program to MK).

\section{References}

1 Lazo M, Hernaez R, Eberhardt MS, et al. Prevalence of nonalcoholic fatty liver disease in the United States: the third national health and nutrition examination survey, 1988-1994. Am J Epidemiol 2013;178(1):38-45

2 Blachier M, Leleu H, Peck-Radosavljevic M, Valla DC, RoudotThoraval F. The burden of liver disease in Europe: a review of available epidemiological data. J Hepatol 2013;58(3):593-608

3 Vernon G, Baranova A, Younossi ZM. Systematic review: the epidemiology and natural history of non-alcoholic fatty liver disease and non-alcoholic steatohepatitis in adults. Aliment Pharmacol Ther 2011;34(3):274-285

4 Williams CD, Stengel J, Asike MI, et al. Prevalence of nonalcoholic fatty liver disease and nonalcoholic steatohepatitis among a largely middle-aged population utilizing ultrasound and liver biopsy: a prospective study. Gastroenterology 2011;140(1): 124-131

5 Chalasani N, Younossi Z, Lavine JE, et al; American Gastroenterological Association; American Association for the Study of Liver Diseases; American College of Gastroenterologyh. The diagnosis and management of non-alcoholic fatty liver disease: practice guideline by the American Gastroenterological Association, American Association for the Study of Liver Diseases, and American College of Gastroenterology. Gastroenterology 2012;142(7): 1592-1609

6 Anstee QM, Targher G, Day CP. Progression of NAFLD to diabetes mellitus, cardiovascular disease or cirrhosis. Nat Rev Gastroenterol Hepatol 2013;10(6):330-344

7 Cusi K. Role of obesity and lipotoxicity in the development of nonalcoholic steatohepatitis: pathophysiology and clinical implications. Gastroenterology 2012;142(4):711-725, e6

8 Sanyal AJ, Brunt EM, Kleiner DE, et al. Endpoints and clinical trial design for nonalcoholic steatohepatitis. Hepatology 2011;54(1): 344-353

9 Sookoian S, Pirola CJ. Meta-analysis of the influence of I148M variant of patatin-like phospholipase domain containing 3 gene (PNPLA3) on the susceptibility and histological severity of nonalcoholic fatty liver disease. Hepatology 2011;53(6):1883-1894

10 Zechner R, Zimmermann R, Eichmann TO, et al. FAT SIGNALSlipases and lipolysis in lipid metabolism and signaling. Cell Metab 2012;15(3):279-291

11 Huang Y, He S, Li JZ, et al. A feed-forward loop amplifies nutritional regulation of PNPLA3. Proc Natl Acad Sci U S A 2010;107(17): 7892-7897

12 Romeo S, Kozlitina J, Xing C, et al. Genetic variation in PNPLA3 confers susceptibility to nonalcoholic fatty liver disease. Nat Genet 2008;40(12):1461-1465
13 Mohanty SR, Troy TN, Huo D, O'Brien BL, Jensen DM, Hart J. Influence of ethnicity on histological differences in non-alcoholic fatty liver disease. J Hepatol 2009;50(4):797-804

14 Weston SR, Leyden W, Murphy R, et al. Racial and ethnic distribution of nonalcoholic fatty liver in persons with newly diagnosed chronic liver disease. Hepatology 2005;41(2):372-379

15 Browning JD, Szczepaniak LS, Dobbins R, et al. Prevalence of hepatic steatosis in an urban population in the United States: impact of ethnicity. Hepatology 2004;40(6):1387-1395

16 Yuan X, Waterworth D, Perry JR, et al. Population-based genomewide association studies reveal six loci influencing plasma levels of liver enzymes. Am J Hum Genet 2008;83(4):520-528

17 Chambers JC, Zhang W, Sehmi J, et al; Alcohol Genome-wide Association (AlcGen) Consortium; Diabetes Genetics Replication and Meta-analyses (DIAGRAM+) Study; Genetic Investigation of Anthropometric Traits (GIANT) Consortium; Global Lipids Genetics Consortium; Genetics of Liver Disease (GOLD) Consortium; International Consortium for Blood Pressure (ICBP-GWAS); Metaanalyses of Glucose and Insulin-Related Traits Consortium (MAGIC). Genome-wide association study identifies loci influencing concentrations of liver enzymes in plasma. Nat Genet 2011; 43(11):1131-1138

18 Kotronen A, Johansson LE, Johansson LM, et al. A common variant in PNPLA3, which encodes adiponutrin, is associated with liver fat content in humans. Diabetologia 2009;52(6):1056-1060

19 Speliotes EK, Yerges-Armstrong LM, Wu J, et al; NASH CRN; GIANT Consortium; MAGIC Investigators; GOLD Consortium. Genomewide association analysis identifies variants associated with nonalcoholic fatty liver disease that have distinct effects on metabolic traits. PLoS Genet 2011;7(3):e1001324

20 Sookoian S, Castaño GO, Burgueño AL, Gianotti TF, Rosselli MS, Pirola CJ. A nonsynonymous gene variant in the adiponutrin gene is associated with nonalcoholic fatty liver disease severity. J Lipid Res 2009;50(10):2111-2116

21 Valenti L, Al-Serri A, Daly AK, et al. Homozygosity for the patatinlike phospholipase-3/adiponutrin I148M polymorphism influences liver fibrosis in patients with nonalcoholic fatty liver disease. Hepatology 2010;51(4):1209-1217

22 Rotman Y, Koh C, Zmuda JM, Kleiner DE, Liang TJ; NASH CRN. The association of genetic variability in patatin-like phospholipase domain-containing protein 3 (PNPLA3) with histological severity of nonalcoholic fatty liver disease. Hepatology 2010;52(3): 894-903

23 Anstee QM, Darlay R, Leathart J, et al. A candidate-gene approach to validation of genetic modifier association using a large cohort with histologically characterized non-alcoholic fatty liver disease. J Hepatol 2013;55(1):S46

24 Krawczyk M, Müllenbach R, Weber SN, Zimmer V, Lammert F. Genome-wide association studies and genetic risk assessment of liver diseases. Nat Rev Gastroenterol Hepatol 2010;7(12):669-681

25 Krawczyk M, Grünhage F, Zimmer V, Lammert F. Variant adiponutrin (PNPLA3) represents a common fibrosis risk gene: non-invasive elastography-based study in chronic liver disease. J Hepatol 2011;55(2):299-306

26 Kawaguchi T, Sumida Y, Umemura A, et al; Japan Study Group of Nonalcoholic Fatty Liver Disease; Takeshi Okanoue. Genetic polymorphisms of the human PNPLA3 gene are strongly associated with severity of non-alcoholic fatty liver disease in Japanese. PLoS ONE 2012;7(6):e38322

27 Kitamoto T, Kitamoto A, Yoneda M, et al. Genome-wide scan revealed that polymorphisms in the PNPLA3, SAMM50, and PARVB genes are associated with development and progression of nonalcoholic fatty liver disease in Japan. Hum Genet 2013;132(7): 783-792

28 Tian C, Stokowski RP, Kershenobich D, Ballinger DG, Hinds DA. Variant in PNPLA3 is associated with alcoholic liver disease. Nat Genet 2010;42(1):21-23 
29 Stickel F, Buch S, Lau K, et al. Genetic variation in the PNPLA3 gene is associated with alcoholic liver injury in Caucasians. Hepatology 2011;53(1):86-95

30 Trépo E, Gustot T, Degré D, et al. Common polymorphism in the PNPLA3/adiponutrin gene confers higher risk of cirrhosis and liver damage in alcoholic liver disease. J Hepatol 2011;55(4):906-912

31 Müller T, Buch S, Berg T, Hampe J, Stickel F. Distinct, alcoholmodulated effects of PNPLA3 genotype on progression of chronic hepatitis C. J Hepatol 2011;55(3):732-733

32 Cai T, Dufour JF, Muellhaupt B, et al; Swiss Hepatitis C Cohort Study Group. Viral genotype-specific role of PNPLA3, PPARG, MTTP, and IL28B in hepatitis C virus-associated steatosis. J Hepatol 2011; 55(3):529-535

33 Valenti L, Rumi M, Galmozzi E, et al. Patatin-like phospholipase domain-containing 3 I148M polymorphism, steatosis, and liver damage in chronic hepatitis C. Hepatology 2011;53(3):791-799

34 Trépo E, Pradat P, Potthoff A, et al. Impact of patatin-like phospholipase-3 (rs738409 C>G) polymorphism on fibrosis progression and steatosis in chronic hepatitis C. Hepatology 2011;54(1):60-69

35 Zampino R, Coppola N, Cirillo G, et al. Abdominal fat interacts with PNPLA3 I148M, but not with the APOC3 variant in the pathogenesis of liver steatosis in chronic hepatitis C. JViral Hepat 2013;20(8): 517-523

36 Vigano M, Valenti L, Lampertico P, et al. Patatin-like phospholipase domain-containing 3 I148M affects liver steatosis in patients with chronic hepatitis B. Hepatology 2013

37 Trépo E, Guyot E, Ganne-Carrie N, et al. PNPLA3 (rs738409 C>G) is a common risk variant associated with hepatocellular carcinoma in alcoholic cirrhosis. Hepatology 2012;55(4):1307-1308

38 Burza MA, Pirazzi C, Maglio C, et al. PNPLA3 I148M (rs738409) genetic variant is associated with hepatocellular carcinoma in obese individuals. Dig Liver Dis 2012;44(12):1037-1041

39 Hassan MM, Kaseb A, Etzel CJ, et al. Genetic variation in the PNPLA3 gene and hepatocellular carcinoma in USA: risk and prognosis prediction. Mol Carcinog 2013

40 Nischalke HD, Berger C, Luda C, et al. The PNPLA3 rs738409 148M/ $M$ genotype is a risk factor for liver cancer in alcoholic cirrhosis but shows no or weak association in hepatitis C cirrhosis. PLoS ONE 2011;6(11):e27087

41 Takeuchi Y, Ikeda F, Moritou Y, et al. The impact of patatin-like phospholipase domain-containing protein 3 polymorphism on hepatocellular carcinoma prognosis. J Gastroenterol 2013;48(3): 405-412

42 Liu YL, Patman G, Leathart J, et al. Carriage of PNPLA3 I148M is associated with an increases risk of non-alcoholic fatty liver disease associated hepatocellular carcinoma. J Hepatol 2013; 58(1):S516

43 do O NT, Eurich D, Trautwein C, Neuhaus P, Neumann UP, Wasmuth HE. The common I148. M variant of PNPLA3 does not predict fibrosis progression after liver transplantation for hepatitis $C$. Hepatology 2011;54(4):1483-1484

44 Finkenstedt A, Auer C, Glodny B, et al. PNPLA3 rs738409-G in recipients of liver transplants is a risk factor for graft steatosis. Clin Gastroenterol Hepatol 2013; [Epub ahead of print]

45 Valenti L, Alisi A, Galmozzi E, et al. I148M patatin-like phospholipase domain-containing 3 gene variant and severity of pediatric nonalcoholic fatty liver disease. Hepatology 2010;52(4): $1274-1280$

46 Santoro N, Kursawe R, D'Adamo E, et al. A common variant in the patatin-like phospholipase 3 gene (PNPLA3) is associated with fatty liver disease in obese children and adolescents. Hepatology 2010;52(4):1281-1290

47 Lin YC, Chang PF, Hu FC, Yang WS, Chang MH, Ni YH. A common variant in the PNPLA3 gene is a risk factor for non-alcoholic fatty liver disease in obese Taiwanese children. J Pediatr 2011;158(5): 740-744

48 Larrieta-Carrasco E, León-Mimila P, Villarreal-Molina T, et al. Association of the I148M/PNPLA3 variant with elevated alanine transaminase levels in normal-weight and overweight/obese Mexican children. Gene 2013;520(2):185-188

49 Krawczyk M, Maier IB, Liebe R, Lammert F, Bergheim I. The common adiponutrin (PNPLA3) variant is associated with subclinical liver injury already in young age: analysis of a cohort of paediatric patients. J Hepatol 2013;58(Suppl 1):S559-S560

50 Marzuillo P, Grandone A, Perrone L, Miraglia Del Giudice E. Weight loss allows the dissection of the interaction between abdominal fat and PNPLA3 (adiponutrin) in the liver damage of obese children. J Hepatol 2013

51 Romeo S, Sentinelli F, Cambuli VM, et al. The 148M allele of the PNPLA3 gene is associated with indices of liver damage early in life. J Hepatol 2010;53(2):335-338

52 Palasciano G, Moschetta A, Palmieri VO, Grattagliano I, Iacobellis G, Portincasa P. Non-alcoholic fatty liver disease in the metabolic syndrome. Curr Pharm Des 2007;13(21):2193-2198

53 Krarup NT, Grarup N, Banasik K, et al. The PNPLA3 rs738409 Gallele associates with reduced fasting serum triglyceride and serum cholesterol in Danes with impaired glucose regulation. PLoS ONE 2012;7(7):e40376

54 Krawczyk M, Grünhage F, Mahler M, Tirziu S, Acalovschi M, Lammert F. The common adiponutrin variant p.I148M does not confer gallstone risk but affects fasting glucose and triglyceride levels. J Physiol Pharmacol 2011;62(3):369-375

55 Rembeck K, Maglio C, Lagging M, et al; NORDynamIC group. PNPLA 3 I148M genetic variant associates with insulin resistance and baseline viral load in HCV genotype 2 but not in genotype 3 infection. BMC Med Genet 2012;13:82

56 Palmer CN, Maglio C, Pirazzi C, et al. Paradoxical lower serum triglyceride levels and higher type 2 diabetes mellitus susceptibility in obese individuals with the PNPLA3 148M variant. PLoS ONE 2012;7(6):e39362

57 Pirazzi C, Adiels M, Burza MA, et al. Patatin-like phospholipase domain-containing 3 (PNPLA3) I148M (rs738409) affects hepatic VLDL secretion in humans and in vitro. J Hepatol 2012;57(6): 1276-1282

58 Kantartzis K, Peter A, Machicao F, et al. Dissociation between fatty liver and insulin resistance in humans carrying a variant of the patatin-like phospholipase 3 gene. Diabetes 2009;58(11): 2616-2623

59 Speliotes EK, Butler JL, Palmer CD, Voight BF, Hirschhorn JN; GIANT Consortium; MIGen Consortium; NASH CRN. PNPLA3 variants specifically confer increased risk for histologic nonalcoholic fatty liver disease but not metabolic disease. Hepatology 2010;52(3): 904-912

60 Angelico F, Del Ben M, Conti R, et al. Insulin resistance, the metabolic syndrome, and nonalcoholic fatty liver disease. J Clin Endocrinol Metab 2005;90(3):1578-1582

61 Farese RV Jr, Zechner R, Newgard CB, Walther TC. The problem of establishing relationships between hepatic steatosis and hepatic insulin resistance. Cell Metab 2012;15(5):570-573

62 Chen W, Chang B, Li L, Chan L. Patatin-like phospholipase domaincontaining 3/adiponutrin deficiency in mice is not associated with fatty liver disease. Hepatology 2010;52(3):1134-1142

63 Basantani MK, Sitnick MT, Cai L, et al. Pnpla3/Adiponutrin deficiency in mice does not contribute to fatty liver disease or metabolic syndrome. J Lipid Res 2011;52(2):318-329

64 Kumari M, Schoiswohl G, Chitraju C, et al. Adiponutrin functions as a nutritionally regulated lysophosphatidic acid acyltransferase. Cell Metab 2012;15(5):691-702

65 Huang Y, Cohen JC, Hobbs HH. Expression and characterization of a PNPLA3 protein isoform (I148M) associated with nonalcoholic fatty liver disease. J Biol Chem 2011;286(43):37085-37093

66 Li JZ, Huang Y, Karaman R, et al. Chronic overexpression of PNPLA3I148M in mouse liver causes hepatic steatosis. J Clin Invest 2012;122(11):4130-4144 
67 Greenberg AS, Coleman RA, Kraemer FB, et al. The role of lipid droplets in metabolic disease in rodents and humans. J Clin Invest 2011;121(6):2102-2110

68 Browning JD, Cohen JC, Hobbs HH. Patatin-like phospholipase domain-containing 3 and the pathogenesis and progression of pediatric nonalcoholic fatty liver disease. Hepatology 2010;52(4): 1189-1192

69 Ratziu V, Cadranel JF, Serfaty L, et al. A survey of patterns of practice and perception of NAFLD in a large sample of practicing gastroenterologists in France. J Hepatol 2012;57(2):376-383

70 Krawczyk M, Bonfrate L, Portincasa P. Nonalcoholic fatty liver disease. Best Pract Res Clin Gastroenterol 2010;24(5):695-708

71 Festi D, Schiumerini R, Marzi L, et al. Review article: the diagnosis of non-alcoholic fatty liver disease - availability and accuracy of non-invasive methods. Aliment Pharmacol Ther 2013;37(4): 392-400

72 Ludwig J, Viggiano TR, McGill DB, Oh BJ. Nonalcoholic steatohepatitis: Mayo Clinic experiences with a hitherto unnamed disease. Mayo Clin Proc 1980;55(7):434-438

73 Mirnezami R, Nicholson J, Darzi A. Preparing for precision medicine. N Engl J Med 2012;366(6):489-491

74 Valenti L, Dongiovanni P, Ginanni Corradini S, Burza MA, Romeo S. PNPLA3 I148M variant and hepatocellular carcinoma: a common genetic variant for a rare disease. Dig Liver Dis 2013;45(8): 619-624

75 Sevastianova K, Kotronen A, Gastaldelli A, et al. Genetic variation in PNPLA3 (adiponutrin) confers sensitivity to weight loss-induced decrease in liver fat in humans. Am J Clin Nutr 2011;94(1): 104-111
76 Calamita G, Portincasa P. Present and future therapeutic strategies in non-alcoholic fatty liver disease. Expert Opin Ther Targets 2007; 11(9):1231-1249

77 Petit JM, Guiu B, Masson D, et al. Specifically PNPLA3-mediated accumulation of liver fat in obese patients with type 2 diabetes. J Clin Endocrinol Metab 2010;95(12):E430-E436

78 Goran MI, Walker R, Le KA, et al. Effects of PNPLA3 on liver fat and metabolic profile in Hispanic children and adolescents. Diabetes 2010;59(12):3127-3130

79 Cox AJ, Wing MR, Carr JJ, et al. Association of PNPLA3 SNP rs738409 with liver density in African Americans with type 2 diabetes mellitus. Diabetes Metab 2011;37(5):452-455

80 Li Y, Xing C, Tian Z, Ku HC. Genetic variant I148M in PNPLA3 is associated with the ultrasonography-determined steatosis degree in a Chinese population. BMC Med Genet 2012;13:113

81 Viganò M, Valenti L, Lampertico P, et al. Patatin-like phospholipase domain-containing 3 I148M affects liver steatosis in patients with chronic hepatitis B. Hepatology 2013

82 Corradini SG, Burza MA, Molinaro A, Romeo S. Patatin-like phospholipase domain containing 3 sequence variant and hepatocellular carcinoma. Hepatology 2011;53(5):1776, author reply 1777

83 Falleti E, Fabris C, Cmet S, et al. PNPLA3 rs738409C/G polymorphism in cirrhosis: relationship with the aetiology of liver disease and hepatocellular carcinoma occurrence. Liver Int 2011;31(8): 1137-1143

84 Guyot E, Sutton A, Rufat P, et al. PNPLA3 rs738409, hepatocellular carcinoma occurrence and risk model prediction in patients with cirrhosis. J Hepatol 2013;58(2):312-318 